\title{
Doubling up on function: dual-specificity tyrosine- regulated kinase 1A (DYRK1A) in B cell acute lymphoblastic leukemia
}

\author{
Jung-Hyun Kim, ${ }^{1}$ Liping Li, ${ }^{1}$ and Linda M.S. Resar ${ }^{1,2,3}$ \\ 'Department of Medicine, Division of Hematology, ${ }^{2}$ Departments of Oncology and Pathology, and Institute of Cellular Engineering, and ${ }^{3}$ Pathobiology, Human Cenetics, and Cellular and Molecular \\ Medicine Graduate Programs, The Johns Hopkins University School of Medicine, Baltimore, Maryland, USA.
}

\begin{abstract}
DYRK1A, the dual-specificity kinase, is again doubling up on function, as reported by Bhansali, Rammohan, and colleagues in this issue of the $J C I$. DYRIK1A is an evolutionarily conserved protein kinase with dual specificity; it adds phosphates to serine/threonine residues of diverse regulatory proteins and activates its own function by autophosphorylating a critical tyrosine at position 321 in the activation loop. Bhansali, Rammohan, and colleagues investigated B cell acute lymphoblastic leukemia (B-ALL) in individuals with Down syndrome (DS) and in children with leukemia characterized by aneuploidy. The study revealed a DYRK1A/FOX01 and STAT3 signaling pathway in B-ALL that could be targeted pharmacologically, thus opening the door to therapeutic strategies for patients with leukemia with or without DS.
\end{abstract}

\section{DYRK1A protein kinases}

Kinases comprise a large class of eukaryotic proteins that evolved to regulate key cellular processes by chemically adding phosphates to modulate protein function. DYRK1A (dual-specificity tyrosine-regulated kinase $\underline{1 \mathrm{~A}}$ ) is among a conserved family of CMGC kinases (named by the first letter of the family members, including cyclin-dependent kinases, mitogen-activated protein kinases, glycogen synthase kinase 3, and, CDK-like kinases) (1). On the basis of structure and function, DYRK kinases are designated class I (DYRK1A, DYRK1B) or class II (DYRK2, DYRK3, DYRK4). DYRK1A is considered to have dual specificity because it phosphorylates diverse regulatory proteins at serine/threonine residues and autophosphorylates its own activation loop to enhance its activity (1). DYRK1A has been intensely investigated, given its positioning on the Down syndrome (DS) critical region of chromosome 21 (HSA21) $(1,2)$, and was later linked to autism and $\mathrm{Alz}$ heimer's disease $(1,3-5)$. Mice that have heterozygous Dyrk1a deficiency recapitulate many of the neurodevelopmental phenotypes observed in DS (1). DS is also associated with a predisposition to cancer, including B cell acute lymphoblastic leukemia (B-ALL), although the mechanisms underlying these seemingly disparate pathologies have remained elusive until now $(2,6)$. As reported in this issue of the JCI, Bhansali, Rammohan, and coauthors' work on DYRK1A sheds light on why individuals with DS develop B-ALL (2). This study also reveals signaling pathways that occur not only in B-ALL in DS, but also in B-ALL with HSA21 polyploidy. Thus, in addition to dual specificity, DYRK1A function, ironically, has dual roles in B-ALL. Notably, the DYRK1A signaling pathway also provides a promising therapeutic target.

Related Article: https://doi.org/10.1172/JCI135937

Conflict of interest: The authors have declared that no conflict of interest exists.

\section{DYRK1A and leukemia}

Studies identifying therapeutic targets in B-ALL are critical because B-ALL is the leading cause of cancer death in children (7-11). Although multidrug therapies have led to cure rates that approach $90 \%$, outcomes for children with relapse remain poor (9). Even those who achieve cures are at risk for toxicity from therapy that can result in complications including diabetes, obesity, and vascular disease. Importantly, children with DS and B-ALL have worse outcomes compared with children without DS (11-14). DS is a relatively common genetic disorder causing pathologic neurodevelopment $(3,5)$. Most frequently, DS is caused by chromosomal nondisjunction during meiotic cell division, resulting in an extra copy (trisomy) of chromosome 21. Children with DS are predisposed to acute megakaryocytic leukemia, which is associated with mutant GATA1 and DYRK1A overexpression (15). In DS megakaryocytic leukemia, DYRK1A dysregulates the nuclear factor of activated $\mathrm{T}$ cell (NFAT) transcription factors, which normally function in megakaryopoiesis (15). Compared with megakaryocytic leukemia, the incidence of B-ALL is even higher in DS, although B-ALL is more heterogenous genetically and includes loss-of-function molecular lesions that inactivate IKZFI and PAX5, cause CRLF2 overexpression, and activate JAK2 (11).

Prior research revealed that DYRK1A is dispensable for myeloid development but required for normal lymphopoiesis, where it targets cyclin D3 for degradation, allowing lymphocytes to become quiescent and differentiate (Figure 1A) (16). The gene encoding high-mobility group $\mathrm{N}$ (HMGN) protein is also positioned within HSA21 and thought to contribute to leukemogenesis by fostering what are known as "poised" or "bivalent promoters" to alter gene expression. HMGN protein also 
A Normal diploid state

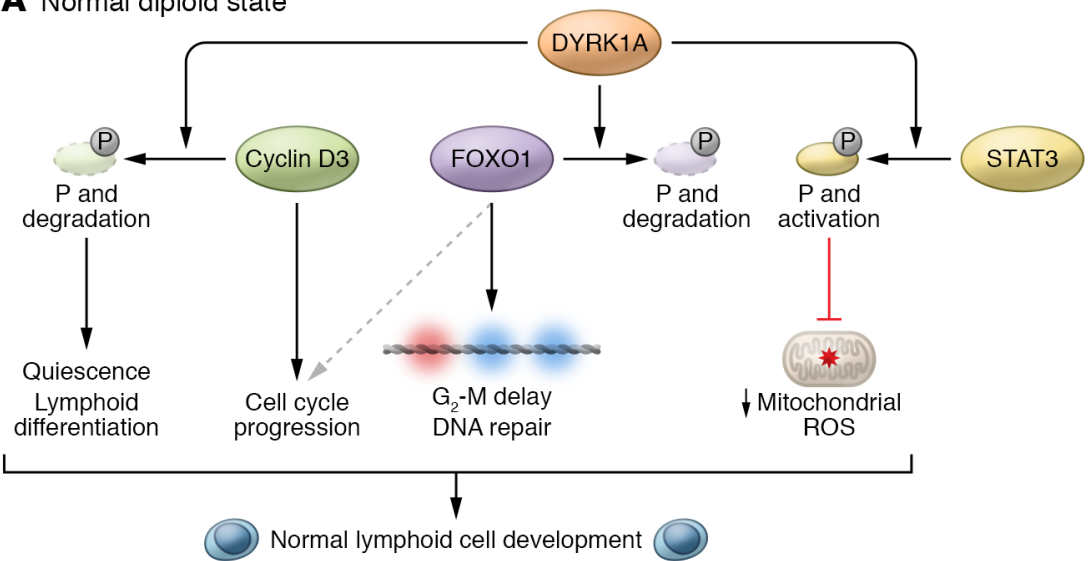

B Trisomy 21, HSA21 polyploidy
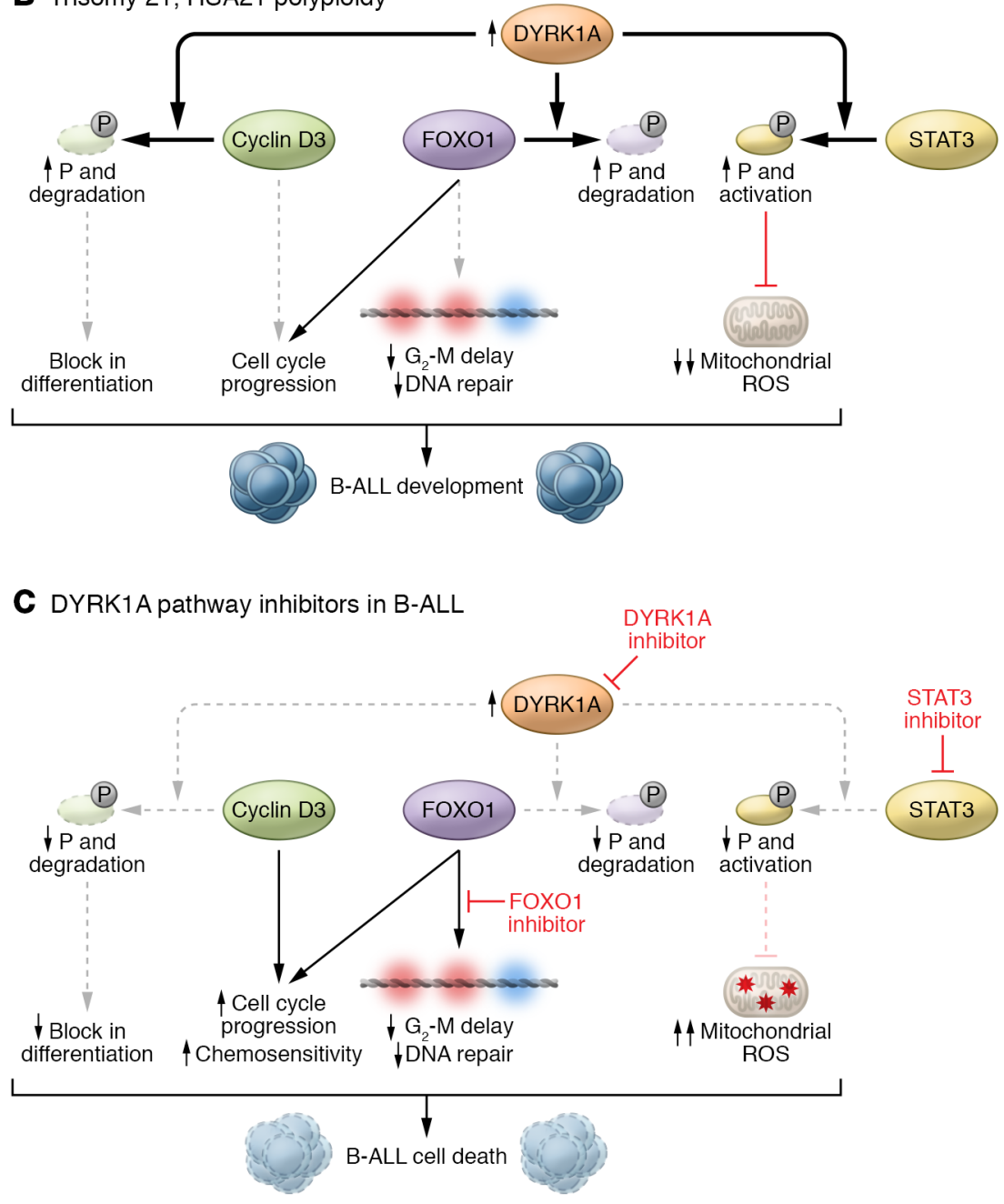

marks critical B progenitor genes with histones for amplification (17-19). Indeed, overexpression of diverse HMG chromatin regulator genes has been linked to aberrant growth and differentiation through epigenetic activation of stem cell transcriptional networks (20-23).

\section{Unique targetable mechanisms} in B-ALL

Bhansali, Rammohan, and colleagues found that, compared with bone marrow mononuclear cells, DYRK1A was overexpressed in diverse B-ALL cell lines and patient samples, including those with poor
Figure 1. Model for DYRK1A/FOXO1 and STAT3 in B-lymphopoiesis and B-ALL. (A) In normal Iymphopoiesis, DYRK1A targets cyclin D3 for degradation, allowing lymphocytes to become quiescent and differentiate. DRYK1A also phosphorylates FOXO1 for degradation, while the remaining FOXO1 enters the nucleus to sense DNA damage, delay the cell cycle, and repair damage. DYRK1A also phosphorylates STAT3, enabling STAT3 to repress mitochondrial ROS (B) Trisomy 21 or DYRK1A polyploidy results in overexpression of DYRK1A and increased phosphorylation of DYRK1A and its substrates (cyclin D3, FOX01, STAT3). In B-ALL, FOXO1 and STAT3 are frequently overexpressed, contributing to even higher levels of phosphorylated FOXO1 and STAT3 when the DYRK1A copy number is increased. The resulting disruptions in cell-cycle progression and decreases in mitochondrial ROS promote leukemogenesis. (C) Inhibition of DYRK1A reduces phosphorylation of DYRK1A and cyclin D3, allowing for cell-cycle progression and chemosensitivity. Inhibition of nuclear FOXO1 also facilitates cell-cycle progression, while blocking repair, thereby fostering chemosensitivity. Blocking STAT3 increases mitochondrial $\mathrm{ROS}$, which could promote B-ALL cell death. prognostic features such as $B C R-A B L$ translocations (Philadelphia chromosome). Because Dyrk1a alone failed to confer clonal expansion in murine B cells in vitro, Bhansali, Rammohan, and colleagues tested whether catalytically active DYRK1 was required for leukemogenesis through a set 
of detailed and elegant experiments. In a murine B-ALL model driven by $B C R-A B L$, the authors deleted Dyrk1a after detecting circulating leukemic cells. Strikingly, even heterozygous Dyrk1a deficiency prevented leukemic cell survival in mice, and, notably, hematopoiesis was normal in the setting of heterozygous Dyrk1a. To evaluate the therapeutic efficacy of targeting DYRK1A, they treated human B-ALL cell lines with DYRK1A inhibitors (EHT 1610 and others), demonstrating dose-dependent decreases in cell numbers. Effects were most pronounced in cells with trisomy 21 or a hyperdiploid status. The researchers also showed synergy of EHT 1610 with multiple chemotherapeutic agents in B-ALL cell lines, particularly in leukemic cells harboring trisomy 21 (2).

To elucidate mechanistic underpinnings, detailed and well-controlled phosphoproteomic studies were performed in murine pre-B cells with genetic deletion of Dyrk1a, revealing that DYRK1A phosphorylated proteins that regulate cell-cycle progression, transcription, and differentiation, including cyclin D3, the forkhead box protein $\mathrm{O} 1$ (FOXO1) transcription factor, and STAT3 (2). FOXO1, which had been implicated in B lymphopoiesis (24), was phosphorylated by DYRK1A at serine 329 (murine Ser326), leading to FOXO1 degradation in the cytoplasm (2) (Figure 1A). Conversely, DYRK1A inhibition promoted nuclear accumulation of FOXO1 (2). In prior studies, FOXO1 was found to sense DNA damage and delay the $\mathrm{G}_{2}-\mathrm{M}$ transition to activate DNA damage response genes, such as Gadd45, thus allowing for repair (25). To assess this pathway, a selective inhibitor of FOXO1 DNA binding (AS1842856) was used in murine pre-B cells, revealing a partial rescue of the proliferation defect and $\mathrm{G}_{2}-\mathrm{M}$ delay induced by DYRK1A blockade. The FOXO1 target Gadd45 was also increased by DYRK1A inhibition, but not when FOXO1 DNA binding was blocked. Intriguingly, FOXO1 protein levels were increased in B-ALL, suggesting a role for FOXO1 in leukemogenesis (2).

To define FOXO1 function in B-ALL (Figure 1B), nuclear levels of FOXO1 were measured after DYRK1A blockade. Nuclear FOXO1 increased relative to levels in normal pre-B cells after DYRK1A inhibition, suggesting that DYRK1A promotes FOXO1 degradation. B-ALL cell lines were also highly sensitive to inhibition of nuclear FOXO1 function compared with murine pre-B cells, particularly those with increased DYRK1A copy numbers. Further, DYRK1A and FOXO1 inhibition (EHT 1610, AS1842856) were synergistic in B-ALL and associated with increased levels of DNA damage, as evidenced by the biomarker $\gamma$-H2AX. Thus, while DYRK1A inhibition increases nuclear FOXO1 and the DNA damage response, this effect was blocked by combination therapy with DYRK1A and FOXO1 inhibitors (Figure 1C). Inhibition of both DYRK1A and FOXO1 led to further decreases in leukemic burdens in diverse murine ALL models compared with DYRK1A inhibitor alone. Importantly, after treating normal mice with DYRK1A or FOXO1 inhibitors, hematologic toxicity was limited to effects on lymphopoiesis (2).

Bhansali, Rammohan, and co-authors sought to investigate the functional interplay between DYRK1A and STAT3, since phosphoproteomic studies identified STAT3 as a potential DYRK1A target, and STAT3 overexpression is associated with worse outcomes in B-ALL, including aggressive forms harboring the BCR-ABL translocations. In addition, B-ALL cell lines with hyperactive JAK/STAT signaling were exquisitely sensitive to DYRK1A inhibition (2). Inhibiting DYRK1A decreased phosphorylation of STAT3 at serine 727, which had been implicated in JAK/STAT signaling and mitochondrial ROS. Expression of STAT3 phosphomimetic mutants resulted in a decrease in mitochondrial ROS, consistent with a protective role for STAT3 in leukemic cells. Bhansali, Rammohan, and co-investigators went on to test a selective STAT3 inhibitor (C188-9) and found that growth of B-ALL cells with hyperactive JAK/STAT signaling was impaired, whereas proliferation of B-ALL cells lacking JAK/ STAT activation was unaffected (2).

\section{Remaining questions and translation}

In summary, this expansive body of work identifies FOXO1 and STAT3 as key factors regulated by DYRK1A in murine pre-B lymphoid cells and B-ALL, most notably in B-ALL blasts harboring extra copies of DYRK1A. Bhansali, Rammohan, and co-authors also identified other putative DYRK1A substrates in pre-B lymphocytes, some of which are likely to contribute to leukemogenesis and normal lymphopoiesis, although further studies will be needed to dissect their roles in each context. Nonetheless, this study illuminates actionable DYRK1A substrates in B-leukemogenesis. Of note, clinical trials are ongoing with JAK/STAT inhibitors (ruxolitinib) in B-ALL with hyperactive JAK/ STAT signaling (BCR-ABL translocated or Philadelphia chromosome-like ALL) and are showing promising results. Studies are warranted to determine whether therapy targeting DYRK1A and FOXO1 enhances the efficacy of JAK/STAT3 inhibitors in these aggressive forms of B-ALL. Moreover, the work of Bhansali, Rammohan, and colleagues (2) beautifully exemplifies the serendipity of science - research focused on B-ALL in DS revealed therapeutic targets relevant to other forms of B-ALL. Thus, while further studies are needed to test whether these results can be safely translated to patients, these findings have the potential to improve outcomes for children with DS as well as others who have B-ALL along with HSA21 polyploidy.

\section{Acknowledgments}

LMSR was supported in part by NIH grants (R01 CA232741, R01 HL145780, R01 DK102943, and R01 HL143818), Alex's Lemonade Stand Foundation, a RALLY Foundation for Childhood Cancer Research Award, the 2020-MSCRFD-5425 Maryland Stem Cell Research Fund, the Hopkins Alleghany Health Network Cancer Research Award, and a Hopkins COVID19 Research Grant.

Address correspondence to: Linda M.S. Resar, 720 Rutland Avenue, Ross Research Building, Room 1015, Baltimore, Maryland 21205-2109, USA. Phone: 410.614.0712; Email:1resar@jhmi.edu.

1. Fernández-Martínez $\mathrm{P}$, Zahonero $\mathrm{C}$, SánchezGómez P. DYRK1A: the double-edged kinase as a protagonist in cell growth and tumorigenesis. Mol Cell Oncol. 2015;2(1):e970048.

2. Bhansali RS, et al. DYRK1A regulates B cell acute lymphoblastic leukemia through phosphorylation of FOXO1 and STAT3. J Clin Invest. 2021;131(1):e135937.

3. Abbassi R, Johns TG, Kassiou M, Munoz L. DYRK1A in neurodegeneration and cancer: Molecular basis and clinical implications. Pharmacol Ther. 2015;151:87-98.

4. Dang T, et al. Autism-associated Dyrk1a trun- 
cation mutants impair neuronal dendritic and spine growth and interfere with postnatal cortical development. Mol Psychiatry. 2018;23(3):747-758.

5. Wiseman FK, et al. A genetic cause of Alzheimer disease: mechanistic insights from Down syndrome. Nat Rev Neurosci. 2015;16(9):564-574

6. Hasle H, et al. Risks of leukaemia and solid tumours in individuals with Down's syndrome. Lancet. 2000;355(9199):165-169.

7. Hunger SP, et al. Improved survival for children and adolescents with acute lymphoblastic leukemia between 1990 and 2005: a report from the children's oncology group. JClin Oncol. 2012;30(14):1663-1669.

8. Hunger SP, et al. Children's Oncology Group's 2013 blueprint for research: acute lymphoblastic leukemia. Pediatr Blood Cancer. 2013;60(6):957-963.

9. Hunger SP, Mullighan CG. Acute lymphoblastic leukemia in children. $N$ Engl J Med. 2015;373(16):1541-1552.

10. Maloney KW, et al. Outcome in children with standard-risk B-cell acute lymphoblastic leukemia: results of Children's Oncology Group Trial AALL0331. J Clin Oncol. 2020;38(6):602-612.

11. Messinger $\mathrm{YH}$, et al. Pediatric acute lymphoblastic leukemia with at $(8 ; 14)$ (q11.2;q32): B-cell disease with a high proportion of Down syndrome: a Children's Oncology Group study. Cancer Genet. 2012;205(9):453-458.

12. Rabin KR, et al. Outcomes in children with Down syndrome (DS) and B-lymphoblastic leukemia (B-ALL): A Children's Oncology Group (COG) report. J Clin Oncol. 2020;38(15_suppl):10510.

13. Buitenkamp TD, et al. Acute lymphoblastic leukemia in children with Down syndrome: a retrospective analysis from the Ponte di Legno study group. Blood. 2014;123(1):70-77.

14. Lee P, Bhansali R, Izraeli S, Hijiya N, Crispino JD. The biology, pathogenesis and clinical aspects of acute lymphoblastic leukemia in children with Down syndrome. Leukemia. 2016;30(9):1816-1823.

15. Malinge $S$, et al. Increased dosage of the chromosome 21 ortholog Dyrk1a promotes megakaryoblastic leukemia in a murine model of Down syndrome. J Clin Invest. 2012;122(3):948-962.

16. Thompson BJ, et al. DYRK1A controls the transition from proliferation to quiescence during lymphoid development by destabilizing cyclin D3. J Exp Med. 2015;212(6):953-970.

17. Lane AA, et al. Triplication of a 21q22 region contributes to $\mathrm{B}$ cell transformation through HMGN1 overexpression and loss of histone H3 Lys27 trimethylation. Nat Genet. 2014;46(6):618-623.
18. Mowery CT, et al. Trisomy of a Down syndrome critical region globally amplifies transcription via HMGN1 overexpression. Cell Rep. 2018;25(7):1898-1911.e5.

19. Cabal-Hierro L, et al. Chromatin accessibility promotes hematopoietic and leukemia stem cell activity. Nat Commun. 2020;11(1):1406.

20. Resar L, Chia L, Xian L. Lessons from the crypt: HMGA1-amping up Wnt for stem cells and tumor progression. Cancer Res. 2018;78(8):1890-1897.

21. Xian L, et al. HMGA1 amplifies Wnt signalling and expands the intestinal stem cell compartment and Paneth cell niche. Nat Commun 2017;8:15008.

22. Schuldenfrei A, et al. HMGA1 drives stem cell, inflammatory pathway, and cell cycle progression genes during lymphoid tumorigenesis. BMC Genomics. 2011;12:549.

23. Shah $\mathrm{SN}$, et al. HMGA1 reprograms somatic cells into pluripotent stem cells by inducing stem cell transcriptional networks. PLoS One. 2012;7(11):e48533.

24. Dengler HS, et al. Distinct functions for the transcription factor Foxo1 at various stages of B cell differentiation. Nat Immunol. 2008;9(12):1388-1398.

25. Huang H, Tindall DJ. Dynamic FoxO transcription factors. JCell Sci. 2007;120(Pt 15):2479-2487. 